\title{
How the optical timing system, the longitudinal diagnostics and the associated feedback systems provide femtosecond stable operation at the FERMI free electron laser
}

\author{
Mario Ferianis, Enrico Allaria, Eugenio Ferrari, Giulio Gaio, Giuseppe Penco, \\ Fabio Rossi, and Marco Veronese \\ Elettra-Sincrotrone Trieste S.C.p.A., Italy \\ (Received 7 August 2015; revised 20 January 2016; accepted 4 February 2016)
}

\begin{abstract}
FERMI, the seeded free electron laser (FEL) in operation in Italy, is providing the User Community with unique fully coherent radiation, in the wavelength range 100-4 nm. FERMI is the first FEL fully synchronized by means of optical fibers. The optical timing system ensures an ultra-stable phase reference to its distributed clients. Several femtosecond longitudinal diagnostics verify the achieved performance; the bunch length monitor (BLM) and the bunch arrival monitor (BAM) will be presented in this paper. Feedback systems play a crucial role to guarantee the needed longterm electron beam stability. A real-time infrastructure allows shot-to-shot communication between front-end computers and the servers. Orbit feedbacks are useful in machine tuning, whereas longitudinal feedbacks control electron energy, compression and arrival time. A flexible software framework allows a rapid implementation of heterogeneous multiinput-multi-output (MIMO) longitudinal loops simply by selecting the appropriate sensors and actuators.
\end{abstract}

Keywords: feedback system; femtosecond; longitudinal diagnostics; optical timing

\section{Introduction}

FERMI is a seeded free electron laser (FEL), routinely providing the User Community with unique state-of-art coherent radiation, in the wavelength range $100-4 \mathrm{~nm}^{[1,2]}$.

In this paper, after an introduction to the seeded FEL scheme, we will present the engineering effort implemented to provide the required femtosecond time stability to the electron bunch needed to ensure a stable generation of the coherent radiation, in short to make it a user facility. Among others systems, to reach the ambitious project objectives FERMI relies on the optical timing system, the singleshot longitudinal diagnostics and the associated feedback systems.

The optical timing system ${ }^{[3]}$ is the backbone infrastructure, ensuring the generation of an ultra-stable phase reference and its distribution to the timing clients which are located at a several hundredth meter distance, throughout the FERMI facility. Having started its commissioning back

Correspondence to: M. Ferianis, Elettra-Sincrotrone Trieste S.C.p.A. di interesse nazionale Strada Statale 14 - km 163, 5 in AREA Science Park 34149 Basovizza, Trieste Italy. Email: mario.ferianis@elettra.eu in August 2009, FERMI has been, worldwide, the first accelerator of a LINAC-based FEL to be fully synchronized by means of an optical fiber-based system. The optical timing system synchronizes with femtosecond accuracy the linear accelerator components, the laser systems and the longitudinal diagnostics.

In order to verify the achieved performance of the timing system during the commissioning and to monitor the effective stability of the whole facility in day-by-day operation, several longitudinal diagnostic instruments with femtosecond precision have been developed and installed in FERMI. These instruments provide, in a nondestructive way for the electron beam, the required femtosecond measurement accuracy at several machine locations, like the relative bunch length monitor (BLM) and the bunch arrival monitor (BAM).

Feedback systems play a crucial role in ensuring high electron beam stability. A real-time infrastructure, based on gigabit Ethernet, allows shot-to-shot communication between different front-end computers, which interface sensors and actuators, and the servers, which execute feedback algorithms. Orbit feedback loops in the LINAC and in the undulator areas are especially useful in machine tuning; 
longitudinal feedback loops, controlling the electron bunch energy, the compression and the time arrival, are essential for long-term stability, by compensating drifts in the radiofrequency (RF) plants, mainly due to temperature variations and component aging. A flexible software framework allows a rapid implementation of heterogeneous multi-input-multioutput (MIMO) longitudinal loops simply by selecting the appropriate sensors and actuators. In this paper, we present the development of the above-mentioned systems as well as the obtained results from field measurement, with special emphasis on the engineering and technological aspects.

\section{The seeded FEL scheme}

FERMI is an FEL, driven by an electron linear accelerator (LINAC); the few picosecond long electron bunches are extracted at $10 \mathrm{~Hz}$ from a $\mathrm{Cu}$ cathode by means of an UV laser and immediately boosted up to about $5 \mathrm{MeV}$ by an RF gun $^{[4]}$. Each bunch is then accelerated up to $1.2-1.5 \mathrm{GeV}$ by $\mathrm{S}$-band RF accelerating structures and along the transport is longitudinally compressed by magnetic chicanes ${ }^{[5]}$ in order to reach a peak current of 500-800 A over few hundreds of femtoseconds. This high brightness beam is then driven in an undulator chain where the FEL process is stimulated and sustained.

FELs are coherent synchrotron light sources; considering the characteristics (spatial and temporal coherence, brightness and spectrum) of the produced radiation, FELs are complementary to synchrotrons. FELs produce by far higher quality radiation, if compared with third-generation Synchrotrons, as it exhibits higher temporal and spatial coherence and a several order of magnitude larger peak brightness ${ }^{[6]}$. Modern x-ray FELs are based on the high-gain FEL concept ${ }^{[7]}$. These systems consist of sending an electron bunch in a sequence of undulators, an array of alternating permanent magnets creating an alternate magnetic field perpendicular to the direction of electron motion ${ }^{[8]}$. The most common FEL configuration for short wavelengths is the so-called self-amplified spontaneous emission (SASE) FEL, a process that amplifies radiation from the electron shot noise ${ }^{[9]}$.

As the electrons proceed downstream the undulators, a synchrotron radiation is produced, which travels with the same group velocity as the bunches, thus interacting with them. As a result a longitudinal density modulation (microbunching) occurs, which results in an even higher coherency of the produced radiation. This gain process continues exponentially, till the so-called saturation is reached after several tens of meters of undulator straight section. Since the radiation process is started from the spontaneous emission which relies on the statistical distribution on the electrons, the SASE pulses are generally characterized by a limited degree of longitudinal coherence and the temporal and spectral properties can change significantly from shot to shot.
FERMI has been conceived on a different scheme, called high-gain harmonic generation $(\mathrm{HGHG})^{[10,11]}$, in which an external laser, named 'seed', interacts with the relativistic electron beam when the latter goes through an undulator, called 'modulator'. The electrons are consequently modulated in energy at the same wavelength of the seed, which is typically the third harmonic of a $\mathrm{Ti}: \mathrm{Sa}$ oscillator. This energy modulation ${ }^{[12]}$ is then converted into a density modulation with strong harmonic components by driving the beam into a dispersive section, i.e., a magnetic chicane. The microbunched electrons are finally injected into a long undulator, called 'radiator', which is tuned to a higher harmonic of the seed wavelength and where the fully coherent FEL emission is exponentially amplified.

\subsection{Critical issue of implementing a seeded FEL}

The beneficial improvements to the quality of the generated radiation due to the seeding process requires an overall higher stability of the whole FEL facility, as both the electron bunch and the seeding laser pulse have to overlap in time and space with a very high accuracy.

The typical length of the FERMI electron bunch at the undulator entrance is several hundreds of femtoseconds at full width half maximum (FWHM), but the region of the bunch to be seeded and with good and homogeneous properties, in terms of emittance, energy spread and peak current, is much smaller: a typical seed laser pulse duration is between 100 and $200 \mathrm{fs}$ (FWHM). The ability to generate electron bunches with a constant energy and current distribution for over 500 fs has been recently demonstrated ${ }^{[13]}$.

In order to guarantee that the seed overlaps with the region of the electron bunch with the required properties, the relative temporal stability between both (jitter and drift) should be better than $100 \mathrm{fs}(\mathrm{rms})^{[14]}$; therefore, the seeding laser needs to be phase locked to the machine phase reference signal to achieve such a goal ${ }^{[15]}$. Also, in the transverse plane, the orbit of the electron bunch and the launch path of the seeding laser have to be co-planar for at least the length of the modulator undulator $(\approx 3 \mathrm{~m})$ within $<20 \mu \mathrm{m}$.

It has been demonstrated that the arrival time jitter of the beam is strongly affected by the stability of the gun and the LINAC (amplitude and phase) ${ }^{[16]}$. Therefore, a feedback on the amplitude and phase stability of all the LINAC accelerating structures (fifteen plants operating at $3 \mathrm{GHz}$ plus one at $12 \mathrm{GHz}$ ) has been implemented ${ }^{[17]}$. On top of an amplitude and phase stabilized LINAC, other feedback loops have been operated to cope with slow drifts of the working point due to slow temperature variations in the accelerator tunnels.

\section{The FERMI optical timing system}

The operation of a seeded FEL requires an ultra-stable timing system $^{[18]}$; in the context of the present work, the term 
timing system indicates all the systems needed to generate and to distribute, over stabilized media, the phase reference signal. The phase reference signal is the clock signal used to synchronize all the accelerator systems needing a femtosecond time stability, like the RF plants, the laser systems and the longitudinal diagnostics. These systems include: the S-band klystrons (feeding both the accelerating and deflecting copper cavities), the X-band klystron used for the longitudinal phase space linearizer; the laser systems: the photoinjector laser, the seed and the user laser. Also the longitudinal diagnostics need an ultra-stable phase reference signal, like the BAM and the RF deflecting cavity.

The frequency of the phase reference has been selected to be equal to $2998.01 \mathrm{MHz}$, which is the frequency of the RF plants (klystrons) feeding the accelerating sections of the LINAC. There are commercially available ultra-low phase noise RF oscillators, which are stable over long time periods. At FERMI, the technical challenge was how to distribute the phase reference signal over the typical distances of an FEL complex $(D \approx 300 \mathrm{~m})$ without spoiling the phase stability of the reference master oscillator (RMO). At the time of FERMI design between 2004 and 2007 it became clear that the high stability requirements of less than $100 \mathrm{fs}$ can not be met with standard copper co-axial lines, unless complex and expensive water temperature stabilization is used. Meanwhile in the accelerator community appeared few solutions for distribution of phase stable reference signals, based on actively stabilized single-mode optical fibers.

At FERMI, according to the jitter sensitivity studies, the required stability (jitter and 8 -h drift) ranges from the $<70 \mathrm{fs}_{\mathrm{RMS}}$ of the LINAC accelerating voltage down to the $<15 \mathrm{fs}_{\mathrm{RMS}}$ of the seed and user lasers. Optical cables ensure a superior electromagnetic noise immunity that has to be traded off with poorer radiation hardness; as expected though at FERMI, radiation losses have shown not to be an issue. Also the superior performance provided by the optical components (optical cross-correlators) used for the stabilization of the links played a fundamental role in our choice. All proposed systems demonstrated, in the laboratory frame, the required level of ultra-low phase noise. We focused our attention on the system developed by LBNL (continuous wave $(C W)$ optical timing in the following $)^{[19]}$ and the one developed ${ }^{[20]}$ by Research Laboratory in Electronics (RLE) at MIT (Pulsed optical timing). At FERMI, an original approach has been adopted ${ }^{[3]}$, the so-called the hybrid optical timing system where both the pulsed and the $C W$ optical timings for the phase reference distribution have been adopted; some of the systems to be synchronized operate in pulsed mode (lasers and diagnostics) whereas others operate in a $\mathrm{CW}$ or quasi-CW mode (LINAC klystrons).

\subsection{The optical timing system layout}

For the FERMI optical timing system, we adopted an asymmetric star topology. The timing hutch is located at the

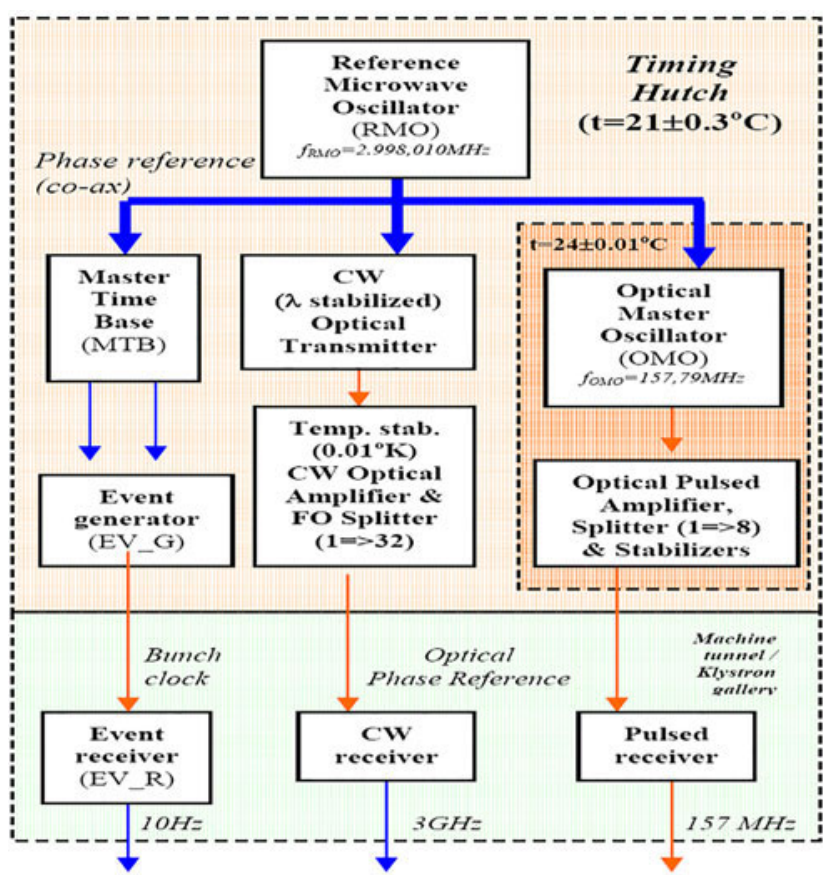

Figure 1. Block diagram of the FERMI timing system.

center star; it is a temperature-controlled $\left(24 \pm 0.2^{\circ} \mathrm{C}\right)$ laboratory located by the accelerator tunnel and close to the photoinjector laser hutch; it houses all the critical equipment of the optical timing system which need to sit close to each other (Figure 1).

For the distribution of the phase reference signal to all the client stations we adopted the Sirocco blown fibers system by Prysmian ${ }^{[21]}$. Each remote station receives either 4 (or 8) fibers in a single bundle. The main remote clients of the timing system are the seeding laser hutch, the various longitudinal diagnostics, distributed along the whole accelerator tunnel, and the user laser hutch $(d \approx 300 \mathrm{~m})$.

\subsection{The pulsed optical timing system}

The pulsed optical timing system is shown in Figure 2. The optical master oscillator (OMO) is a soliton fiber laser ${ }^{[20]}$ working at a repetition rate of $157.790 \mathrm{MHz}$ (S-band 19). Two modules are provided for the phase locking of the OMO to the phase reference signal: a conventional fast photodiode-phase detector (FPD-PD) and an innovative balanced optical micro-wave-phase detector (BOM-PD), designed by the group at MIT ${ }^{[20]}$, specifically engineered for FERMI by Menlo Systems GmbH (Munich, D).

The optical signal of the OMO, amplified by an Er-doped fiber amplifier (EDFA), is split (8 ways) to feed the link stabilization (FLS) units $(\times 6)$. The path between the OMO and each FLS is entirely in fiber; the dispersion compensation ensures the shortest pulses in front of the link crosscorrelators. Each splitter output port provides $\approx 40 \mathrm{~mW}$ of 


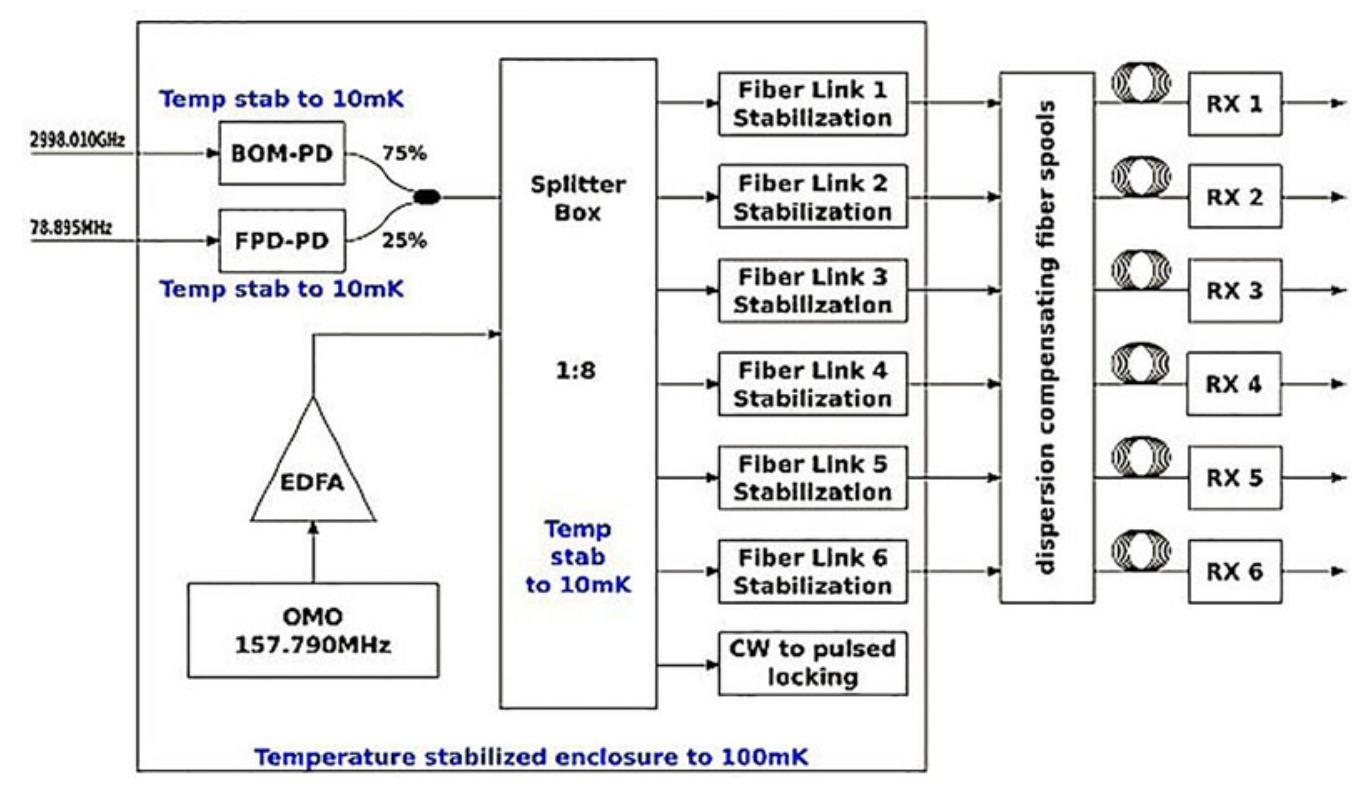

Figure 2. Block diagram of the pulsed optical timing system.

power with an average pulse width of 165 fsFWHM $_{\text {FWd }}$ $30 \mathrm{~nm}$ of bandwidth. The path length variations are actively compensated by the combined action of a piezo-mirror, on the short time scale, and a motorized translation stage, for the drifts.

The system components, up to the FLS units, are installed in the timing hutch in a temperature-controlled box, stable to $0.1 \mathrm{~K}$; the splitter box, inside the same box, is stable down to $10 \mathrm{mK}$. The loop electronics implements a PID controller, interfaced to the control system, driven by the error signal generated from the pulse overlap. An optical receiver is located at each remote end of the stabilized links; it is composed of an electro-optic module, containing the link amplifier used to overcome the losses along the link, and of a passive head where a $10 \%$ reflecting Faraday Rotator Mirror is installed. To synchronize a remote laser oscillator, first direct conversion of the optical pulse train is used for coarse locking, followed by optical techniques, e.g., optical cross-correlation, for precise phase locking.

\subsection{The continuous wave optical timing system}

As previously stated, the relative time (i.e., phase) stability of the different RF plants of the LINAC is of paramount importance for the quality (i.e., low energy spread) of the electron bunch. Therefore, to stabilize the phases and amplitudes of the fifteen $3 \mathrm{GHz}$ RF plants, at FERMI the digital Low Level RF (LLRF) system, developed at LBNL ${ }^{[19]}$, has been adopted. The distribution of the phase reference is accomplished by means of the so-called CW optical links, in which the phase reference signal is encoded on the optical carrier by an amplitude modulation. A new concept has been adopted in this system by measuring the drift of each individual link with femtosecond accuracy and by periodically adding this value as a digital delay in the LLRF loop.

Any moving part has been omitted in order to improve the overall reliability. A single optical amplifier is fitted to the 32-channel sender unit which is feeding the links and the individual Sync Head/Link stabilizer units to drive local LLRF controllers.

\subsection{Characterization of the optical timing system}

The most critical part of the optical timing system is the distribution of the pulsed reference signals used for the synchronization of the lasers systems and for the sampling of the electron bunch in the BAM diagnostics. In the seeded FEL, the timing stability of the seed laser (both short and long term) is as crucial as the timing overlap of the beam with the electron bunch.

The design and implementation of the pulsed subsystem has been carried out trying to optimize the relative timing stability between the endpoints of the links, whereas longterm drifts are compensated with an active beam-based feedback system.

During the commissioning of the pulsed optical timing, the validation of the implemented timing distribution has been carried out by comparing the output of a $150 \mathrm{~m}$ stabilized fiber link to the local optical reference. This total length does not include the dispersion compensating fiber (DCF), but only the single-mode fiber (SMF) of the link. The optical link, installed in the LINAC tunnel, has been configured in loop back mode such that the optical pulses stream has been 


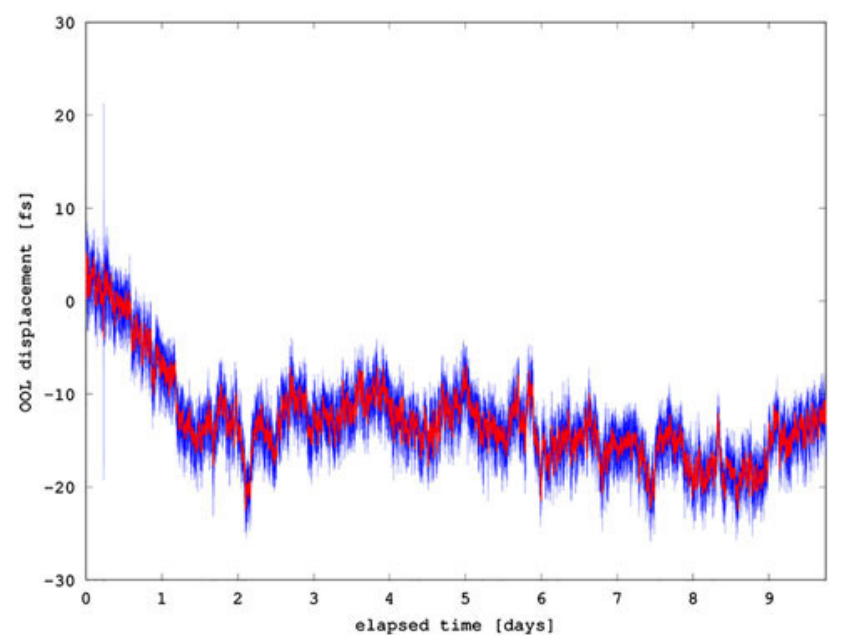

Figure 3. OOL stability measurement of a $150 \mathrm{~m}$ pulsed stabilized link (loopback mode). The residual relative drift between the link output and the local splitter port is equal to $5.3 \mathrm{fs}_{\mathrm{RMS}}$.

made available close to the transmitter end, i.e., in the timing room where the pulsed OMO is installed. An important aspect of this testbed is that the optical fiber was installed in the real accelerator tunnel, with all the EM-noisy RF plants switched on.

The validation consisted in an out-of-loop (OOL) measurement between the stabilized link output and one of the splitter ports, at the star center. The phase comparison between the two signals has been accomplished by means of an optical balanced cross-correlator ${ }^{[22]}$ with a sensitivity of $35 \mathrm{mV} / \mathrm{fs}$, while the temperature around the OOL part of the setup was controlled at $\pm 150 \mathrm{mK}$. In Figure 3, an OOL measurement is presented, with a long-term $5.3 \mathrm{fs}_{\mathrm{RMS}}$ stability over ten days; this residual drift is probably related with polarization changes in the link.

The final characterization of the timing system performance has been carried out by means of an indirect measurement using the BAM diagnostics described later; this kind of verification indeed provides an upper limit of the intrinsic performance of the timing system. This beam-based measurement provides an experimental demonstration of the global stability of FERMI and of its timing system (optical pulsed and optical CW). This measurement shows the relative stability between the pulsed and the $\mathrm{CW}$ reference, the first one used to synchronize the photoinjector laser and the BAM station, the second one used to synchronize the RF plants. In Figure 4, a typical long-term acquisition is presented exhibiting a $30.4 \mathrm{fs}_{\mathrm{RMS}}$. Typical, short-term (50 s) time stability is $\approx 30 \mathrm{fs}_{\mathrm{RMS}}$, while on longer term $(1.6 \mathrm{~h})$, a slow drift is visible and needs to be compensated for. Therefore, to make full use of the timing femtosecond stability, it is necessary to implement a feedback based on the BAMs, as described later on in this paper, extending the timing stability on longer time scales.

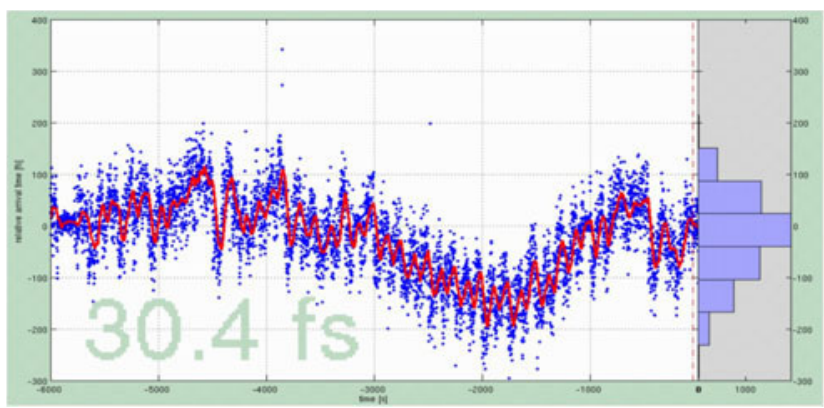

Figure 4. Acquisition showing the typical trend of the arrival time at the BAM station, installed after the first bunch compressor. The feedback based on the arrival time was not active.

\section{The longitudinal diagnostics}

Longitudinal diagnostics are instruments developed to perform measurements in the longitudinal axis of the reference system, i.e., in the direction of the electron motion (also referred to as ' $z$ ' axis). They are fundamental for the commissioning of an FEL facility as the longitudinal axis is one dimension of the so-called $6 \mathrm{D}$ phase space, which completely defines the properties of the electron bunch. In the bunch $6 \mathrm{D}$ phase space, the conjugate variable of the ' $z$ ' axis is the electron energy. So performing measurements on the longitudinal axis discloses the information about the electron bunch energy distribution and the energy spread; particles with different energies have different locations along the bunch. In order to gain a detailed insight on the bunch parameters, so-called slice measurements have been introduced and implemented on FERMI as well. Slice measurement means that we are not only measuring a bunch property referred to the whole bunch envelope, but rather we measure that property for several adjacent slices in the same bunch, each slice being tens of femtoseconds long.

Longitudinal diagnostics for an FEL can be considered as advanced diagnostics as the requirements and associated specifications for these instruments are often very tough and require to address new techniques and physical principles and new components which are typically not needed in thirdgeneration light source diagnostics. As it will be explained later in this paper, often these new techniques are based on electro/optical effects or radiative sources for extracting the information about the electron bunches. Finally, from the end-user point of view, these diagnostics have also to be 'nondestructive' to the beam meaning that when used they shall not disturb the electron bunches. Being nondestructive, these diagnostics can be used while the FEL is ON and are, therefore, ideally suited for generating an error signal to be used in feedback system loops.

At FERMI, several innovative nondestructive longitudinal diagnostics have been engineered and successfully installed on the machine; in this paper, we present the (relative) BLM and the BAM. During the development of these systems, in 


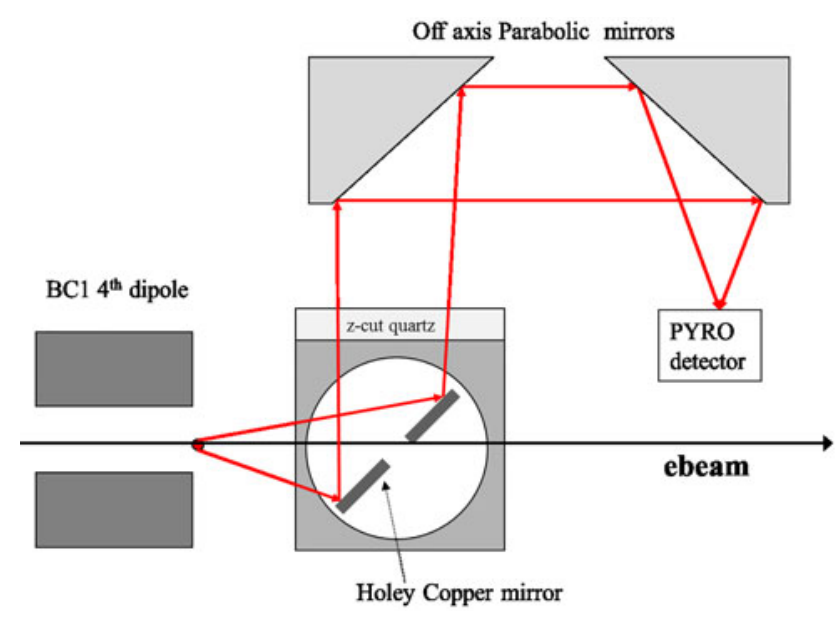

Figure 5. Layout of the BLM.

the framework of the EUROFEL Design Study project ${ }^{[23]}$, we benefit from frequent contacts and suggestions from colleagues at DESY, where these new techniques have been demonstrated for the first time ${ }^{[24,25]}$. To commission, and also to obtain an absolute calibration value for these longitudinal diagnostics, an RF-based technique has also been deployed on FERMI, which is the RF deflecting cavity ${ }^{[26,27]}$.

\subsection{The relative $B L M$}

Both the absolute and the relative bunch length measurements are key parameters to the FERMI commissioning and, today, they are extensively used in everyday operation. The FERMI relative BLM design ${ }^{[28]}$ is based on the LCLS BLM system $^{[29]}$. The first BLM station has been installed downstream the first bunch magnetic chicane (BC1) of FERMI. It is based on the coherent radiations power measurement.

The BLM has been designed to perform relative bunch length measurements in the range of bunch length (flat top current profile) from 5 to $0.15 \mathrm{ps}$, full width (FW). To entirely cover such a broad range of bunch lengths, two coherent radiation sources have been selected. The first source is the coherent synchrotron radiation from the bending magnets of the $\mathrm{BC} 1$ compressor. The second is coherent diffraction radiation from a ceramic gap $^{[30]}$. In this paper, we are only discussing the BLM based on the pyrodetectors, covering the range from 1 to $0.15 \mathrm{ps}$ FW. Ts transfer function is limited at low frequencies by the angular acceptance of the transport system and by the sensitivity of the pyrodetectors.

The layout of the BLM system installed on BC1 at FERMI is shown in Figure 5 (electrons moving left to right). The vacuum chamber is composed of a cube located immediately downstream the last dipole of the BC. It houses a holey mirror that reflects the radiation upwards to a collimating and focusing pair of off-axis parabolic mirrors. The first

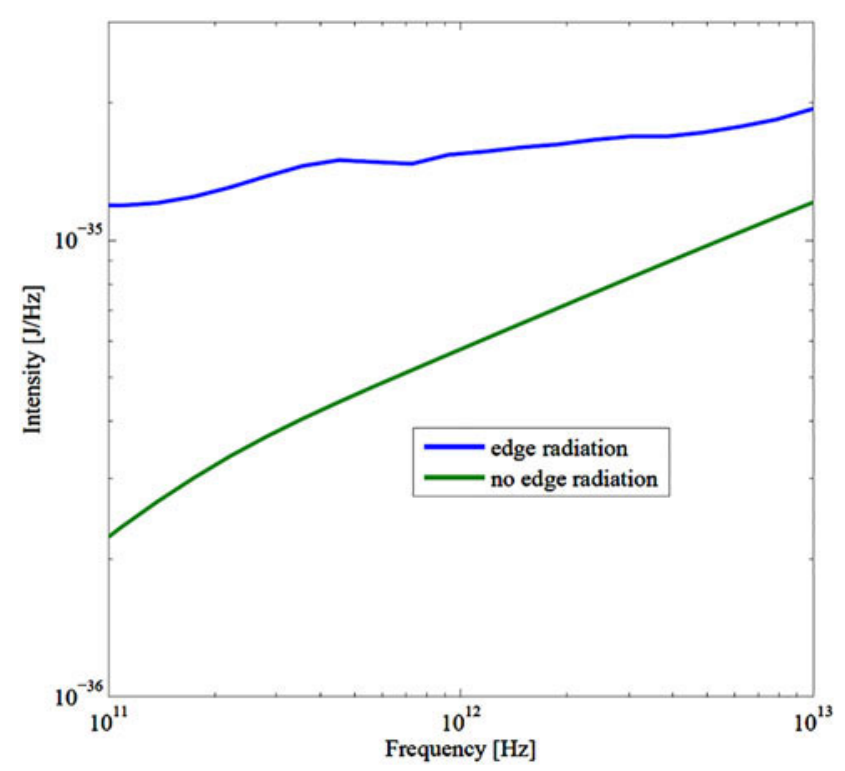

Figure 6. Single electron spectral dependence of the radiation emitted from the fourth dipole of $\mathrm{BC} 1$; the edge radiation (blue) is dominated by the velocity term and the synchrotron radiation (green) is dominated by the acceleration term.

mirror is used to collimate the radiation, while the second is used to focus it onto the pyrodetector. A $z$-cut quartz window is used to separate the vacuum from the atmosphere. A dry air purging enclosure surrounds both the mirrors and the pyrodetector. The gap system is installed immediately downstream and the detection is performed by $3-\mathrm{mm}$-wave diodes with central frequencies around 30,100 and $300 \mathrm{GHz}$, respectively. The diodes are mounted on an optical breadboard, fitted with translation stages to allow varying the distance between gap and diode.

A detailed study of CSR-CER radiation properties has been performed using the Synchrosym code ${ }^{[31]}$ based on the Liénard-Wiechert potentials. The code handles the emission from an arbitrary magnetic field profile and evaluated both velocity and acceleration terms and also include mirror charges, the effect of the third and fourth dipoles and both velocity and acceleration terms, compared with the classical synchrotron radiation emission.

In Figure 6, the single electron spectral dependence of the radiation is shown: at low frequency, the radiation is strongly dominated by edge radiation, while, at higher frequencies, the pure synchrotron radiation component become almost comparable. Moreover, a small but not negligible contribution from radiation coming from the third dipole can also be expected. The optimization of the system included an analysis of the spectral transmission of the whole transport systems. Limitations in the transmission for low frequencies arise from geometrical apertures; longer wavelength radiations are emitted at larger angles, thus apertures typically create low-frequency cutoffs. At high frequencies, the limitations arise from the transmission of the vacuum windows 


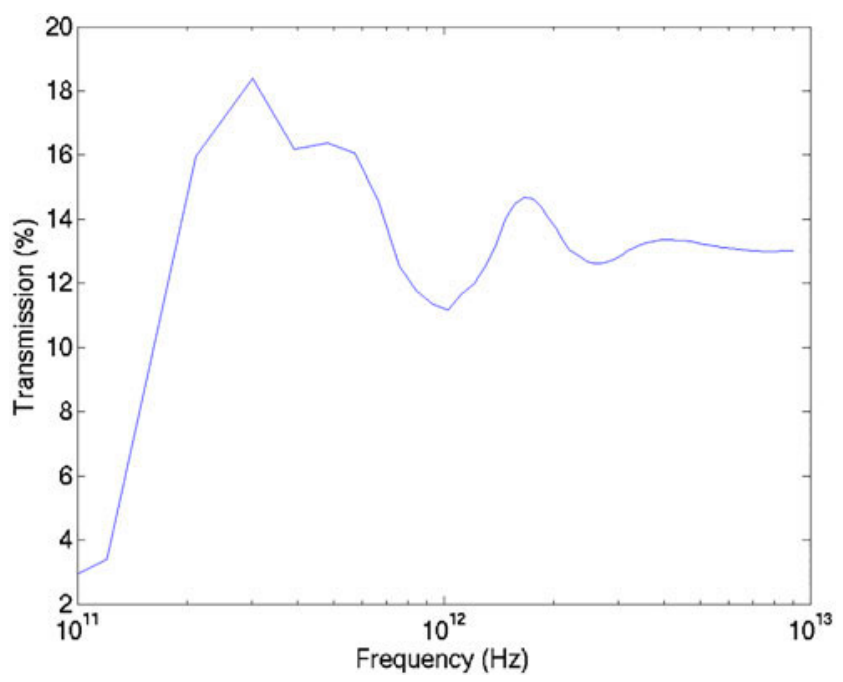

Figure 7. Spectral dependence of the transmission of the optical system.

used to extract the radiation and from water absorption related to humidity in the air. For these reasons we decided to use $z$-cut crystal quartz as the window material which guarantees transmission up to about $3 \mathrm{THz}$ and is transparent in the visible, allowing an optical alignment of the mirrors. To reduce the impact of humidity the window, the mirrors and the detector are separated from the rest of the atmosphere by a sealed enclosure. A dry air purging system is capable to reduce the relative humidity $(\mathrm{RH})$ in the enclosure from an ambient RH of $45 \%$ to a value of about $1 \%$ in less than $20 \mathrm{~min}$. In the range of bunch length so far explored the suppression of humidity lead to an improvement of about $10 \%$ in the total energy transmission of the system.

Figure 7 shows the spectral dependence of the optical transmission of the system at the pyrodetector. A MATLAB code from Loos has been used to optimize the aperture diameter, the distances and the size of the mirrors to allow for optimal radiation collection from $300 \mathrm{GHz}$ to $10 \mathrm{THz}$. The low-frequency cutoff is mainly due to the limited initial angular acceptance caused by mechanical constraints and only partially due to diffraction at the detector.

In Figure 8, the BLM signal acquired while varying the compression factor of $\mathrm{BC} 1$, by changing the phase of the previous LINAC section, is shown; the bending angle has been kept constant. The pyrodetector signal versus phase, shown in Figure 8, becomes detectable only when the bunch becomes short enough, that is at about a bunch length of $1.5 \mathrm{ps}$ FW. About the detection performance, in terms of signal to noise ratio (SNR), the diodes show very good performance, a fact which is important for use in feedback systems. The pyrodetector SNR is $\approx 10 \%_{\text {RMS }}$.

The BLM is based on the detection of coherent radiation whose spectral power depends quadratically on the number of electrons and charge variations affect the output signal. When the BLM is used in a compression feedback these

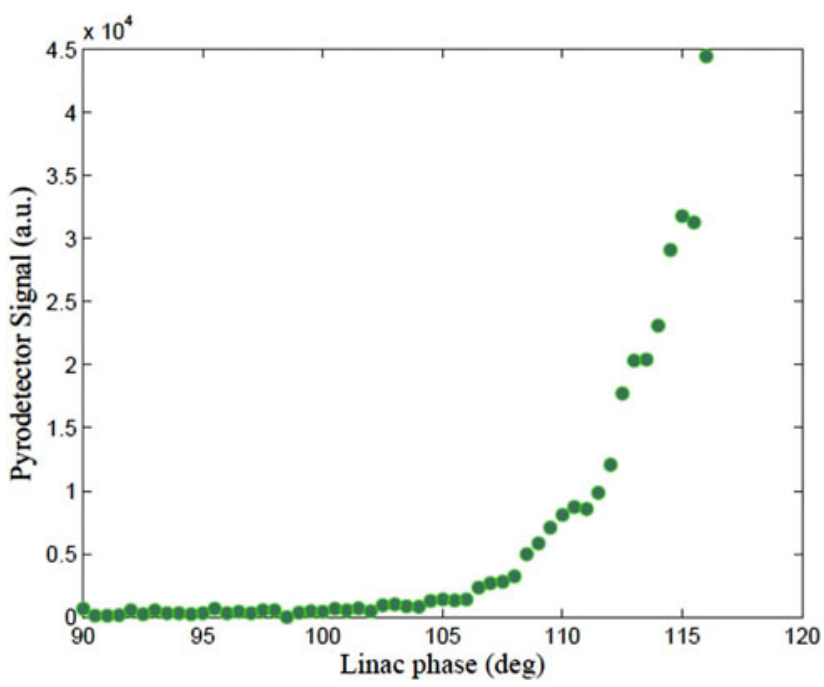

Figure 8. Pyrodetector signal versus LINAC 1 RF phase.

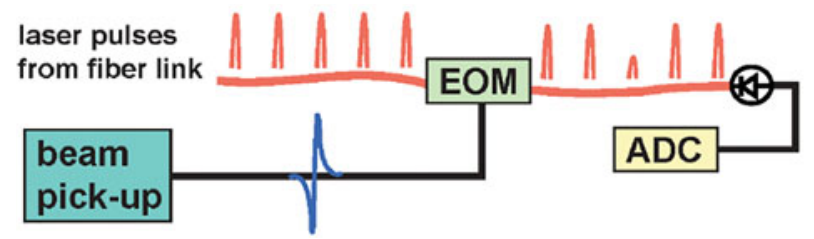

Figure 9. Schematic representation of the $\mathrm{BAM}^{[34]}$.

effects are particularly unwanted and a good short- and longterm charge stability is needed. Short-term (shot-by-shot) charge stability is correlated to the gun RF amplitude and phase fluctuations and to the photocathode (PC) laser energy stability; on Fermi, the charge rms is less then $1 \%$. On the other hand, long-term drifts of the charge are controlled by a dedicated slow charge feedback which has the LH charge monitor as sensor and the PC laser UV variable attenuator as the actuator.

\subsection{The BAM}

The BAM is based on an original idea developed at FLASH/DESY ${ }^{[32]}$ and it has been specifically designed and implemented in-house for FERMI; a detailed description of the FERMI implementation can be found ${ }^{[33]}$.

As schematically shown in Figure 9, the electron bunches produce an RF transient with a steep slope in a high bandwidth Pickup (bandwidth $\geqslant 12.5 \mathrm{GHz}$ ). Highly stable reference laser pulses are delivered to the BAM station via length stabilized pulsed optical links. One of these reference laser pulses interacts with the RF transient in a Mach-Zehnder electro-optical modulator (MZM) with a $12 \mathrm{GHz}$ bandwidth.

In a case of overlap, the arrival time of the electron bunch is encoded in the amplitude of one reference laser pulse. Because of the high phase stability of the pulsed reference 


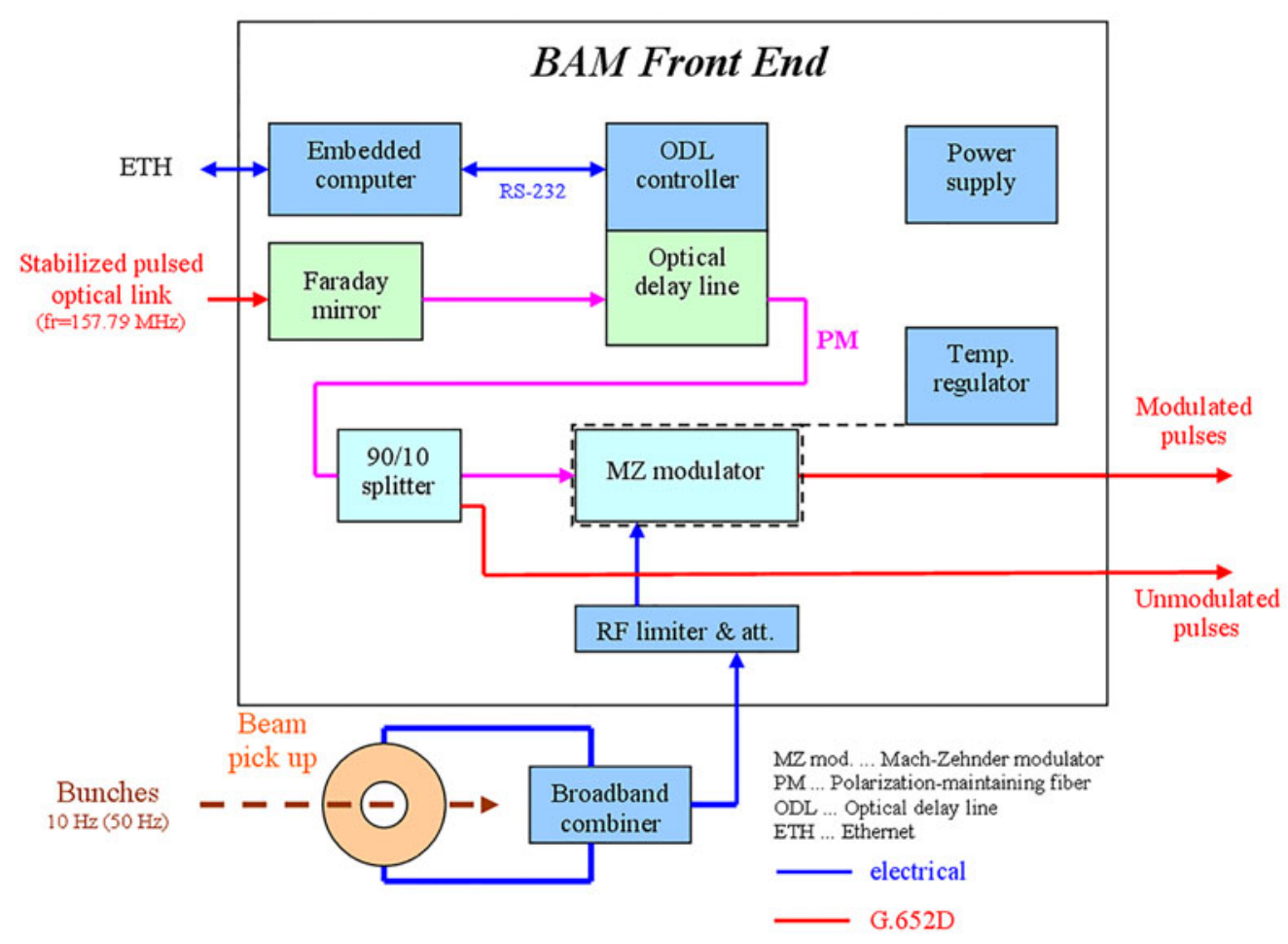

Figure 10. Block diagram of the BAM front end.

distribution, such arrival time measurement is highly stable and reliable. In addition, the measurement is nondestructive to the bunch.

At the operating point, one optical pulse of the optical pulsed reference has to time overlap with the zero crossing of the first RF transient from the pick up (modulating signal).

To effectively amplitude modulate the overlapping optical pulse stream, the MZM has to be DC-biased to operate in quadrature such that all the optical laser pulses are modulated to the half amplitude. By doing so, shot-to-shot electron arrival time fluctuations with respect to the optical reference pulses produce a different modulating voltage at the MZM modulating input. Hence electron arrival time fluctuations produce amplitude variations of the optical pulse.

Several BAM stations have been installed on FERMI; two in the LINAC (at BC1 and at BC2) and one before each of the two FEL straight sections, named FEL1 and FEL2, respectively.

Each BAM station consists of two separate units: the front end, installed in the tunnel close to the beam pick up (to minimize the loss on the electrical signal, maximizing the resolution of the BAM diagnostic), and the back end, installed in the service area for the analog to digital conversion of the amplitude modulated pulses and for further processing.

In the BAM front end, shown in Figure 10, the relative timing between the reference optical pulse and the electron bunches is measured with a typical resolution of $10 \mathrm{fs}$ (with a $500 \mathrm{pC}$ nominal bunch charge). The Faraday mirror, part of the optical timing stabilized link, has to be as close as possible to the MZM to minimize the effect of the timing drifts occurring outside the stabilized link. Given the high loss of the MZM (in the order or $10 \mathrm{~dB}$ ), we decided to leave this fiber interconnection uncompensated from the point of view of the dispersion to avoid excess losses due to the SMF-DCF splicing. The splitter transmits $90 \%$ of the optical power to the MZM, while the not modulated $10 \%$ is used for the extraction of the clock to the ADC, located in the back end.

An optical delay line is used for the calibration of the system; the calibration is performed by sweeping the optical pulse around the zero crossing of the first slope of the pick up signal.

The two ADCs located in the back end acquire the peaks and the baseline points in a 512-sample window around the zero crossing; the effective amplitude of each acquired pulse is given by the difference between the peak and the adjacent baseline sample. The result of the calibration is a curve reporting the modulation index (i.e., the ratio between the amplitude of the modulated pulse to the average amplitude of the not modulated pulses in a suitable time window before the modulating event) versus the optical delay line position.

Since the MZM transfer function is temperature dependent, the modulator is temperature stabilized within $100 \mathrm{mK}$ by a Pulse Width Modulation temperature controller, using a thermoelectric element. The unit is remotely controlled, via Ethernet line, where a micro-controller drives all the elements and provides diagnostic information. 


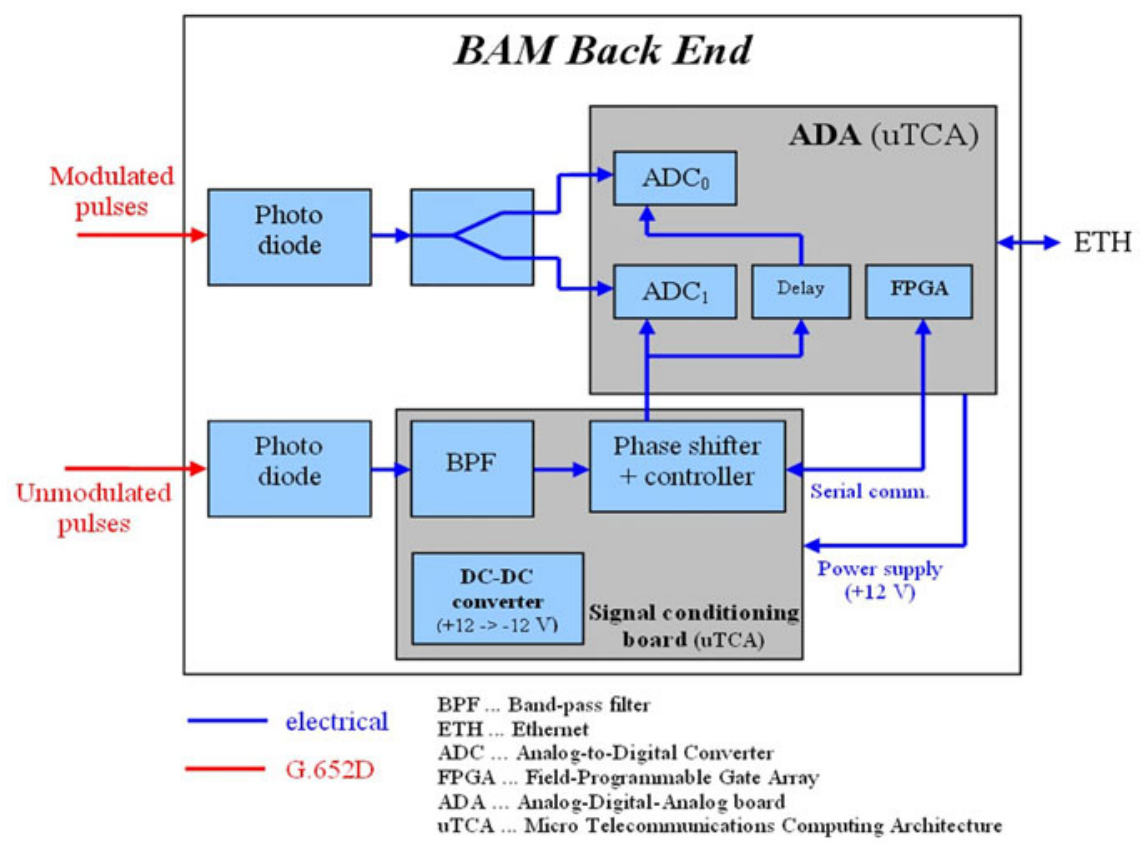

Figure 11. Block diagram of the BAM back end.

The BAM back end (shown in Figure 11) digitizes the modulated pulses using a dedicated analog-digital-analog (ADA) board, based on the $\mu$-TCA platform ${ }^{[34]}$. Besides the ADA board, the BAM back end also includes the signal conditioning board (SCB) which extracts the analog-todigital converter (ADC) clock signal, provides a low-phase noise electrically adjustable phase shifter (based on a passive IQ modulator) and features an automatic gain control for the incoming signal. Two high-speed photodiodes implement optical-electrical conversion.

The modulated pulse signal is split in two and the two outputs are sampled by two 16-bit ADCs working at a rate of $157 \mathrm{MHz}$. The delay between the two ADC sampling times is kept constant (equal to a few hundreds of picoseconds) to acquire both the peak and the baseline of each pulse to get rid of common mode amplitude fluctuations. The back end is also remotely controlled via Ethernet connection (in this case a real-time communication is needed to implement shot-byshot feedbacks) and the ADA board is also in charge to drive the SCB.

The resolution of the BAM depends on the slope of the RF transient, on the amplitude noise of the optical pulses and on the overall stability of the acquisition electronics. The amplitude jitter, normalized in percent of the modulation, has been measured on the not modulated pulses; typical values are in the range $0.13 \%-0.18 \%$.

The resolution of the BAM has been also validated ${ }^{[35]}$ by analyzing the shot-to-shot correlation between two BAM stations.

An upper limit for the resolution of the diagnostic was estimated neglecting possible contributions to time jitter due

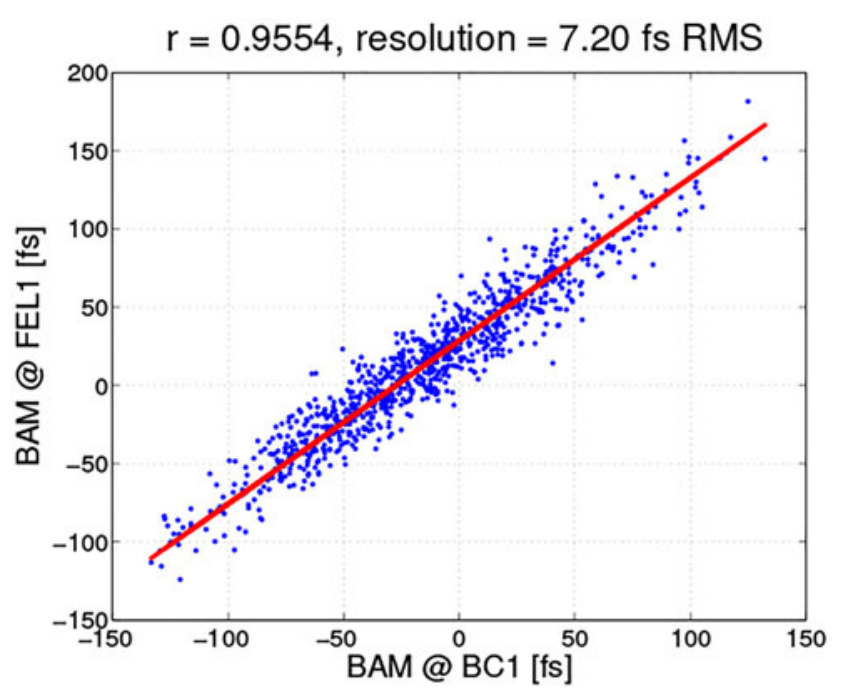

Figure 12. Estimation of the BAM resolution. By splitting in quadrature the contributions of two independent BAM stations, the width of the shot-toshot correlations shows an upper estimation for the resolution in 8 fs RMS (bunch charge $=500 \mathrm{pC}$ ).

to the accelerating sections in between the two diagnostics (installed in two spots of the machine whose distance is roughly $100 \mathrm{~m}$ ) and assuming independent and identical performance of the two stations (such that their contribution may be added in quadrature). These assumptions are reasonable as the correlation coefficient is always greater than $95 \%$. The result obtained over 1000 shots (shown in Figure 12) indicates an upper limit of the BAM resolution lower than 8 fs $_{\text {RMS }}$. 


\section{The longitudinal feedback systems}

Since the beginning of FERMI commissioning (back in 2009-2010), the machine proved to be quite stable in keeping its working point, provided the temperature of the whole facility was kept constant within $\pm 0.5^{\circ} \mathrm{C}$. It is also true that as the experiments to be performed became more and more sophisticated, in terms of FEL pulse generation schemes, and the time span of each experiment extended to several days of 24-h operation, the demands in terms of general machine stability became more stringent. Therefore, dedicated feedback system loops, some based on the longitudinal diagnostics presented above, have been progressively added to the control room work stations, to ensure the required stability.

\subsection{Introduction to FEL feedback systems}

The key factors to keep the FEL radiation output stable are both the transverse and the longitudinal (i.e., temporal) stability at the interaction point between the seed laser pulses and the electron bunches, along the modulator undulator. Besides the uncorrelated shot-to-shot noise (that cannot be reduced by beam-based feedback systems), the main cause of FEL output power fluctuations is related to slow thermal drifts which mainly affect the RF plants and the laser systems. These instabilities have frequencies below $0.1 \mathrm{~Hz}$ and only beam-based feedbacks effectively damp them out.

In order to maximize the feedback efficiency to dump such kind of Noises, all loops run on a shot-to-shot time base. Sensors and actuators interface to PPC VME frontend computers running Linux with real-time extension and managed by kernel modules. The sensor values are shared among the control system computers by means of a shared memory called network reflective memory (NRM). This software communication protocol, based on Gigabit Ethernet and working at Linux device driver level, has been developed to implement a shared memory across the whole control system, allowing computers to communicate in real time.

The NRM topology is a star-like topology network: a CPU master, every $400 \mu \mathrm{s}$ broadcasts an Ethernet jumbo packet $(9 \mathrm{~kb})$ which contains the updated values on the NRM. When the CPU slave receives the master Ethernet packet containing data updates, it replies to the master with a packet containing new values. The data transfer from one CPU to the others takes a maximum of two NRM cycles $(800 \mu \mathrm{s})$. More than two hundred sensors and actuators plus another 20 machine diagnostic parameters are easily monitored and controlled via the NRM. In particular, two frameworks (one controlling transverse position of electron and laser beams and the other controlling longitudinal electron beam dynamics) have been developed on top of the NRM. Once the list of sensors and actuators of a new feedback is defined, the framework deploys a new loop automatically.
The system, which supports MIMO models, relies for the static part of the loop on the measure of the linear relation between the inputs and the outputs, which we call the response matrix $(\mathrm{RM})$. The $\mathrm{RM}$ is calculated by measuring the perturbations on the sensors produced by each actuator involved in the loop: the actuators are driven sequentially to produce a step or a ramp while the sensors are acquired synchronously to the actuator excitation. At the end of the process, the RM resembles a lower triangular matrix where column values correspond to the distortion measured by the sensors due to the actuator kick. The amplitude of the actuator kicks has to be chosen carefully: a too small kick could be confused in the sensor noise while a large one could induce nonlinear effects on the sensor readings.

While the product between the RM and a combination of actuator values (in order to avoid nonlinear effects the values has to be relatively small) could forecast the effects on sensor readings, the multiplication of the inverted RM with the sensor values returns the list of actuator values which cause the current sensor readings. Practically a feedback system, based on the RM, multiplies the inverted RM by the error (difference between the feedback set point and the actual sensor readings) and subtracts the result from actuator values. The RM inversion is carried out through the singular value decomposition (SVD): in the inversion procedure it is possible to define for each sensor/actuator a weight. The singular values, which come out from the SVD, could be individually weighted as well. Cutoff/Tikhonov singular value weighting strategies are directly integrated in the feedback system.

A low pass filter in series with a proportional integral derivative (PID) controller controls the dynamic part of the loop. Actually the controller parameters are tuned by hand. For every feedback the PID is tuned in order to get a step response without overshoot and, at the same time, try to keep the maximum magnitude of the sensitivity function under 'one' across all the loop bandwidth.

All system models involved in feedback systems could be assumed to be equivalent to one delay because the time elapsed from the sensor acquisition to actuator new setpoint reaching is within a feedback period $(20 \mathrm{~ms})$. The closed loop feedback response could be displaced from the theoretical due to errors in the empirical model, the RM. This happens when the number of input-output increases and the actuators are not independent. In this case a cutoff of the singular values or a Tikhonov regularization of the inverted $\mathrm{RM}$ is necessary.

\subsection{FERMI longitudinal feedback systems}

In FERMI, three different beam-based longitudinal feedback loops are active:

- Energy feedback: It keeps constant the energy of the machine, up to a certain point, by reading the horizontal position of the electron beam measured by a beam 


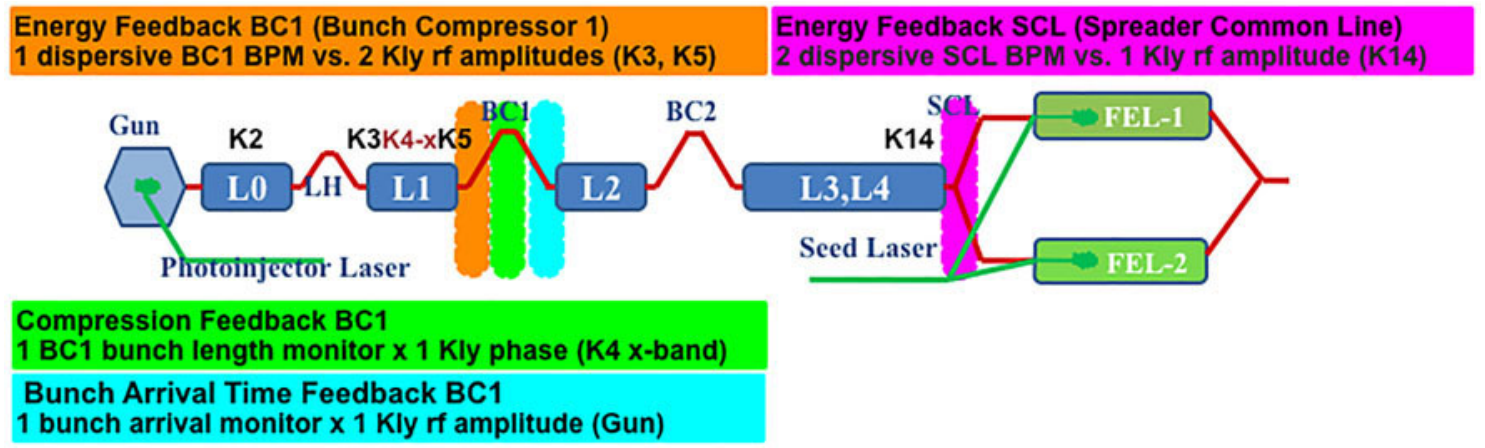

Figure 13. Layout of FERMI longitudinal feedbacks.

position monitor (BPM) in a high dispersive region and by changing the RF amplitude of a klystron acting on a low-level radio-frequency controller (LLRF).

- Compression feedback: It keeps constant the bunch length of the electron bunch measured by the BLM and changing the phase of a klystron by means of the LLRF.

- Bunch arrival time feedback: It keeps constant the arrival time of the electron bunch relative to the reference timing by reading a BAM and changing the RF amplitude of a klystron acting on the LLRF.

While the energy loops are based on transverse diagnostics, the BPMs, the bunch arrival time and the compression loops use the longitudinal ones (Figure 13). Several longitudinal feedback schemes have been exploited during FERMI operation. As most of the electron beam instability sources come from the first bunch compressor $(\mathrm{BC} 1$ and the components before it), a great part of the feedback schemes cover this region. Common sense would suggest gathering the largest number of sensors and actuators in a single feedback (in order to decrease cross-talk effects between not fully independent sensors and actuators). Nevertheless, the sensitivity to RF plant failures of a much more complex control scheme made us split a global longitudinal feedback into a series of equivalent single input-single output feedback loops.

At FERMI, there are two longitudinal diagnostics based feedbacks: the bunch arrival feedback and the compression feedback.

\subsection{Bunch arrival time feedback system}

The bunch arrival feedback acquires the arrival time of the electron bunch in BC01 with respect to the FERMI reference timing and keeps it constant, by changing the RF amplitude of the gun. The feedback is based on the principle that the electron bunch time of flight is very

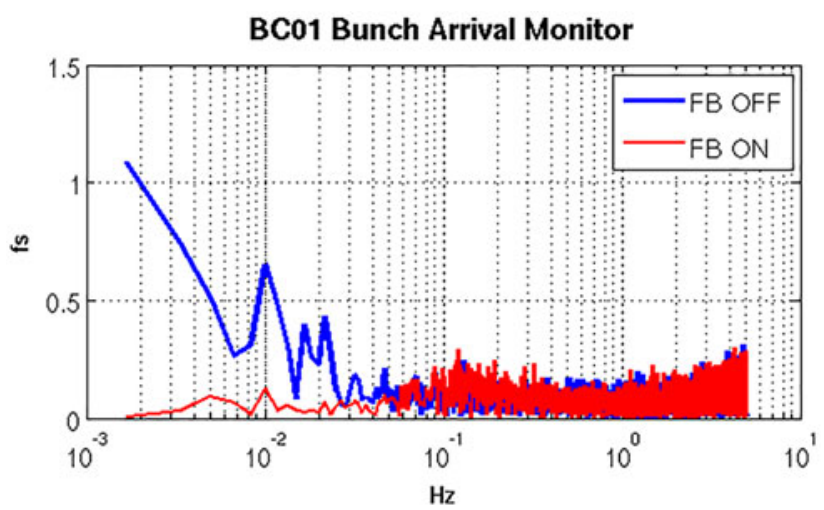

SFEL01 Bunch Arrival Monitor

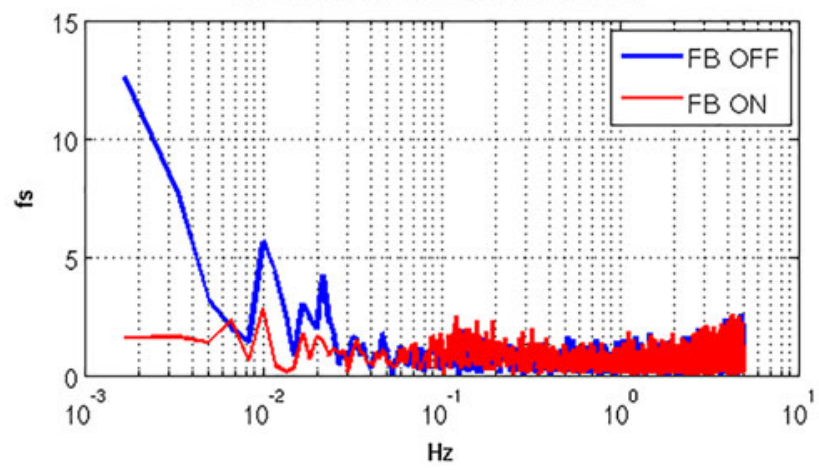

Figure 14. Spectra of the two BAMs with BC1 BAM feedback, OFF and $\mathrm{ON}$.

sensitive to the RF amplitude in the gun because at such a low energy $(<5 \mathrm{MeV})$ electrons are not yet relativistic. In order to evaluate the feedback performance, a second BAM, installed in front of the first modulator undulator acts as an exit of the loop sensor. Actually the bunch arrival feedback is able to dump the arrival time instabilities up to $0.05 \mathrm{~Hz}$ and its effectiveness is also confirmed by the BAM installed in SFEL01 (shown in Figure 14). There is a residual slow timing drift (with a period of about $20 \mathrm{~min}$ ) of about $40 \mathrm{fs}$ peak-to-peak between the two BAMs, probably due to a small part of the system outside temperature compensation. 


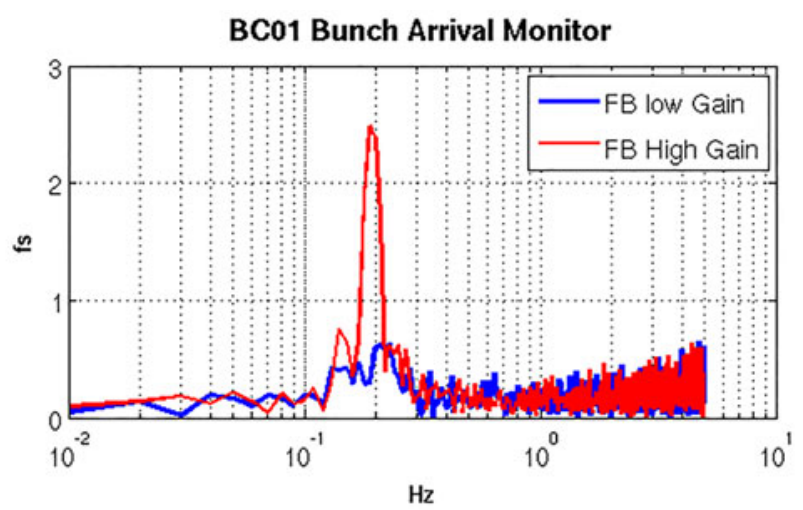

BC01 Energy Monitor

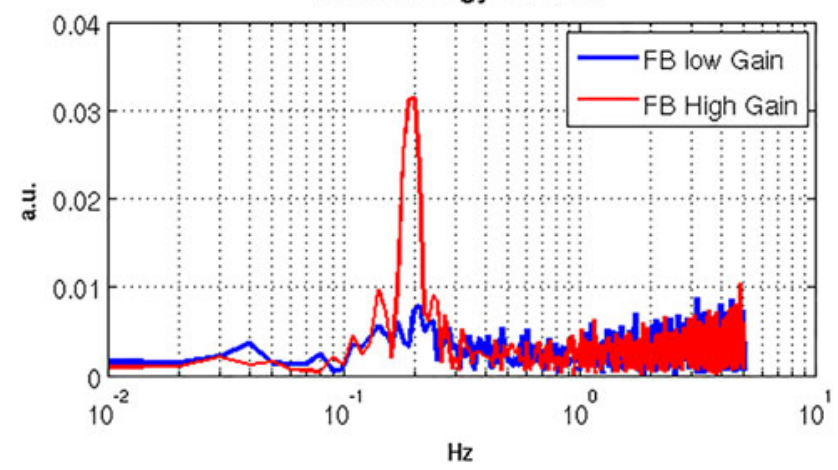

Figure 15. Spectra of the bunch arrival (top) and energy (bottom) sensor feedbacks, with bunch arrival feedback configured with low or high loop gain.

The time of flight of the electron bunch up to $\mathrm{BC} 1$ is also strongly dependent of the electron beam energy. In fact, the first bunch compressor converts energy jitter in arrival time jitter so that a decoupling strategy, based on loop gain fine tuning, has to be adopted to avoid cross-talk between bunch arrival and energy feedback in $\mathrm{BC} 1$ (as shown in Figure 15).

\subsection{The compression factor feedback system}

The bunch compressor is a key component of FERMI. It is a magnetic chicane used to produce high peak current electron bunches. The compression factor is tuned by changing different key parameters: the mechanical angle at the chicane, the dipole current, the off-crest phase shift of the S-band sections of LINAC 01 (see Figure 13) that are powered by RF plants Klystron 3 and Klystron 5. All these are set automatically using dedicated programs. Once the compression factor is set, the compression feedback is switched on.

The feedback loop tunes compression factor, measured as the output of a pyroelectric detector, in a small working range. The correlation of the BLM reading with the FEL output power is normally quite strong, so turning off the compression feedback causes large FEL output power oscillation with a frequency below $0.01 \mathrm{~Hz}$ (Figure 16).

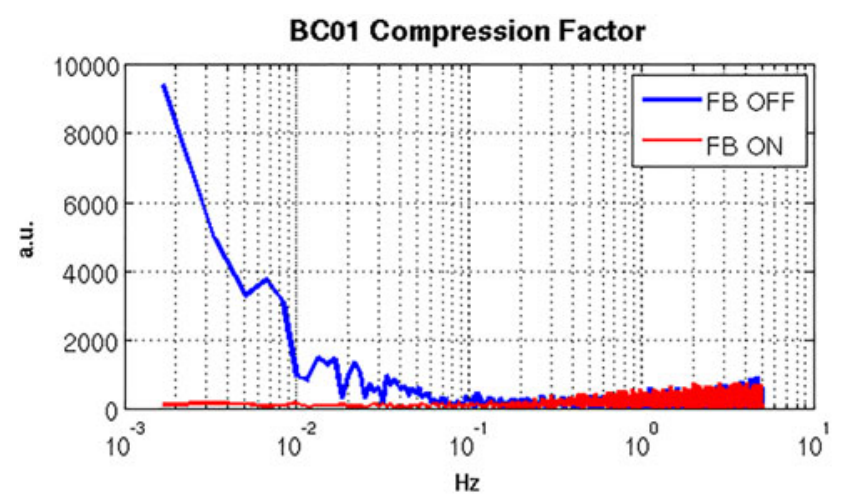

FEL Intensity monitor

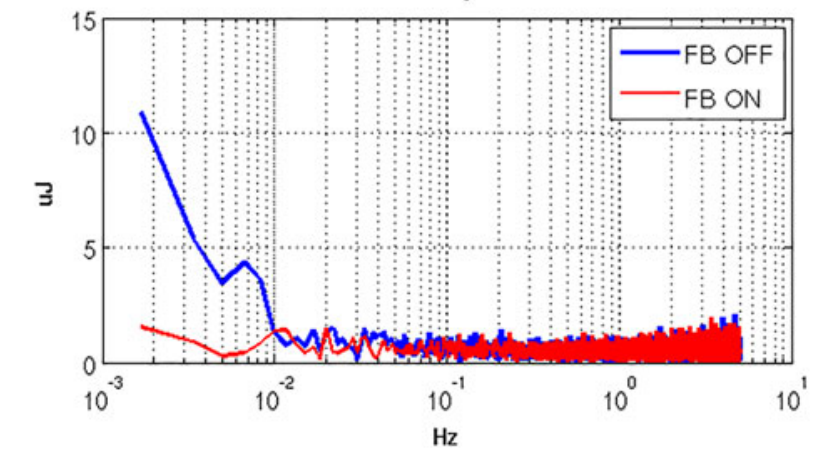

Figure 16. Spectra of the $\mathrm{BC} 1$ compression factor (top) and the FEL output power (bottom) when compression feedback is switched OFF and ON.

\section{FERMI longitudinal phase space characterization}

FERMI is equipped with three RF deflecting cavities $^{[26,27,36-38]}$, one located in the low energy part of the machine ${ }^{[39]}$ and two located at the end of the LINAC ${ }^{[40]}$. These are used to perform destructive beam measurements, i.e., the e-beam is not available for FEL operations during this measurements.

The working principle of the RF deflector is to provide a null deflecting voltage to the bunch centroid and stretches the electrons linearly from the head to the tail of the bunch, when its RF phase is properly set. In this way electrons transverse displacement are correlated with their longitudinal position along the bunch. Coupling the RF deflector with a diagnostic fluorescent screen allows to characterize the bunch current profile and the time-sliced parameters, as the slice emittance. A time-sliced temporal resolution of about $10 \mathrm{fs}$ has been estimated $^{[40]}$. In fact, RF deflectors have been utilized to cross-calibrate the (online) diagnostics described above.

Moreover, as previously mentioned, a detailed knowledge of the longitudinal phase space of the electron bunch (the electron energy distribution as a function of the electron temporal position along the bunch) is of paramount importance to a successful FEL commissioning and operations. At this purpose the beam stretched via the RF deflector can be sent into an energy spectrometer that chromatically disperses electrons in the other (horizontal) transverse plane. 


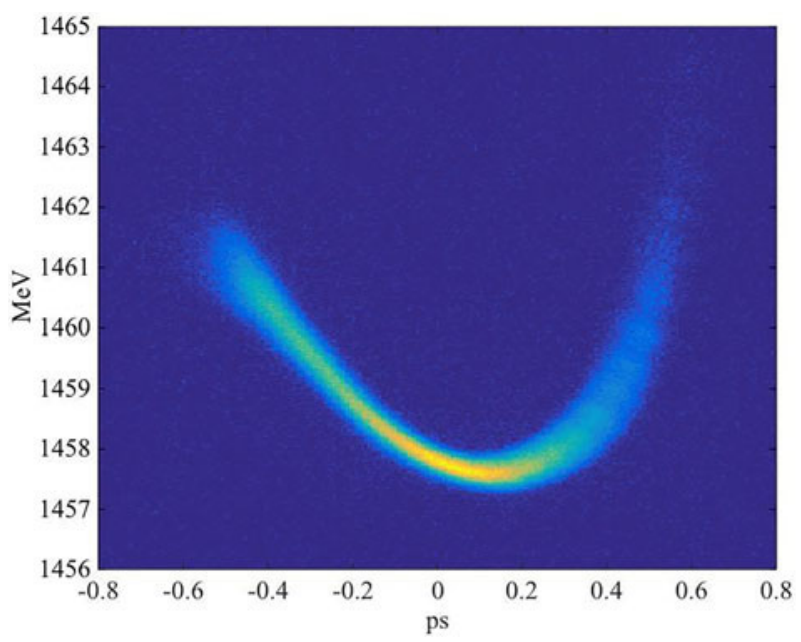

Figure 17. 700 pC-bunch longitudinal phase space imaged on a YAG screen placed in the energy spectrometer at the end of the LINAC; the head of the bunch is on the left.

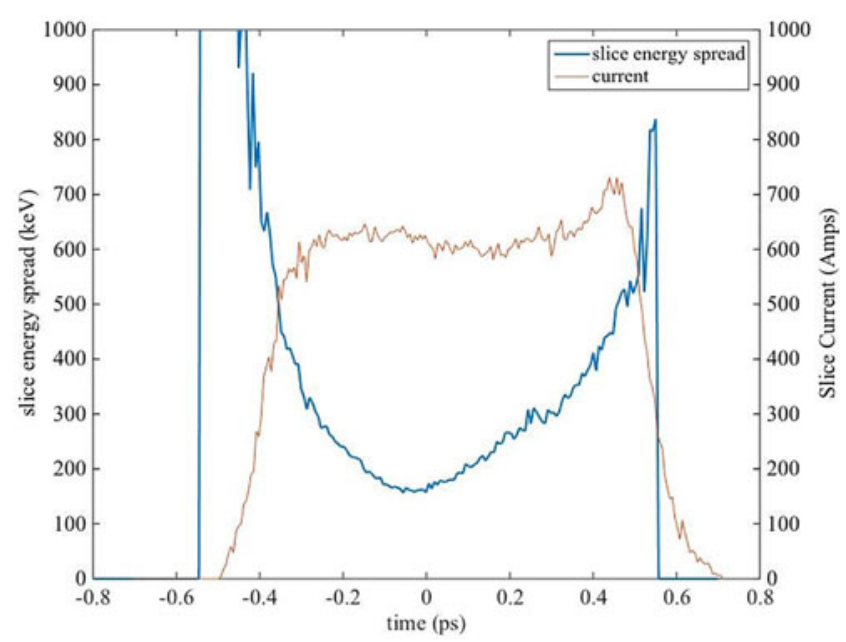

Figure 18. Time-sliced current (red line) and energy spread (blue line) along the bunch obtained from the longitudinal phase space reported in Figure 6.1, relative to a $700 \mathrm{pC}$ bunch. The head of the bunch is on the left.

The bunch longitudinal phase space can then be visualized on a fluorescent screen system (YAG crystal + CCD camera) placed downstream, as shown in Figure 17.

Temporal slicing of the data in the acquired image, i.e., selecting small vertical portions of the longitudinal phase space, provides the current profile (red line in Figure 18) and the uncorrelated energy spread (blue line in Figure 18) along the bunch. Unfortunately, this measurement is destructive and is not available during the FEL operations.

The use of a deflecting cavity after the undulator would permit for a noninvasive and online characterization of the electron beam phase space and FEL induced effects on the electron beam, as currently in use at $\operatorname{LCLS}^{[41]}$.

\section{FERMI operation stabilization using the feedback systems}

As discussed in the introduction, the main advantage of externally seeded FEL configurations, like the one used at FERMI, concern the control of the FEL process. As a result of the seeding the FEL properties are no longer determined by the shot noise and stochastic effects, but only depend on the electron beam and seed laser properties. To really take advantage of this, it is crucial to be able to control both the seed laser and the electron beam with very high accuracy. A good timing system and dedicated feedbacks are needed to keep both beams, as much as possible, stable in time. In addition to independently stabilize the two beams, that need to interact in the modulator, a perfect synchronization between the two has to be guaranteed.

\subsection{Feedback for electron beam stabilization}

Beam properties of the electron beam entering into the undulator and producing the FEL radiation are determined by all systems involved from the generation of the electron at the gun and the following acceleration and manipulation along the LINAC. In addition to the stabilized timing system the use of beam-based feedback is needed for compensating long-term drifts that may not be compensated by locally acting feedbacks. The most used beam-based feedbacks at FERMI are:

- the electron beam orbit feedback;

- the beam energy feedback;

- the beam compression feedback.

\subsection{Feedback for seed laser stabilization}

In order to transfer to the electron beam the coherent energy modulation, the seed laser pulse needs to be superposed in space and time to the electrons within the modulator. At FERMI, the distance between the last available laser optic and the interaction region is of the order of $10 \mathrm{~m}$. Moreover, another $10 \mathrm{~m}$ of optical transport is needed to bring the laser pulse from the laser room to the last injection optics. As a result, keeping micrometer pointing and fstiming stability of the laser is not a trivial task and it may also be spoiled by slow changes related to temperature changes or other mechanical drifts. Several feedback loops have been implemented on FERMI to keep the laser as much stable as possible; these include ${ }^{[42]}$ :

- transverse laser feedback;

- cross-correlation feedback.

\subsection{Feedback e-beam-seed stabilization}

Because some of the electron beam properties (i.e., electron beam energy) change along the single electron bunch with 
time scales of tens of femtoseconds ${ }^{[12]}$, the relative timing between electron bunches and seed laser pulses need to be kept constant with tens of femtoseconds accuracy over several hours or days. Small changes of electron beam properties would indeed affect the FEL performance. In order to compensate these possible drifts between the arrival time of the electron beam and the seed laser in the undulator, a new feedback system has been implemented very recently that reacts to changes of the FEL properties and calculates the amount of timing change to be applied to the machine.

Since a small change of the relative timing in between the electron beam and the seed laser can lead to a small change of the FEL emission wavelength ${ }^{[43]}$ this can be used to correct for drifts in the relative time between electron and seed. Other options are based on measurements of the FEL power and stability to calculate the needed correction and has been shown to be effective in compensating drifts of few tens of femtoseconds over a few days.

\section{Acknowledgments}

The authors would like to thank the FERMI team (including the diagnostics group, the laser group, the control group and the FERMI operators) for constantly assuring the best operating point of FERMI.

They also acknowledge all funding sources that made the FERMI construction and operation possible, among others: the Italian Minister of University and Research (MIUR), the Regione Autonoma Friuli Venezia Giulia, the European Investment Bank (EIB), the European Research Council (ERC) and the European Commission (EC).

\section{References}

1. E. Allaria, R. Appio, L. Badano, W. A. Barletta, S. Bassanese, S. G. Biedron, A. Borga, E. Busetto, D. Castronovo, P. Cinquegrana, S. Cleva, D. Cocco, M. Cornacchia, P. Craievich, I. Cudin, G. D'Auria, M. Dal Forno, M. B. Danailov, R. De Monte, G. De Ninno, P. Delgiusto, A. Demidovich, S. Di Mitri, B. Diviacco, A. Fabris, R. Fabris, W. Fawley, M. Ferianis, E. Ferrari, S. Ferry, L. Froehlich, P. Furlan, G. Gaio, F. Gelmetti, L. Giannessi, M. Giannini, R. Gobessi, R. Ivanov, E. Karantzoulis, M. Lonza, A. Lutman, B. Mahieu, M. Milloch, S. V. Milton, M. Musardo, I. Nikolov, S. Noe, F. Parmigiani, G. Penco, M. Petronio, L. Pivetta, M. Predonzani, F. Rossi, L. Rumiz, A. Salom, C. Scafuri, C. Serpico, P. Sigalotti, S. Spampinati, C. Spezzani, M. Svandrlik, C. Svetina, S. Tazzari, M. Trovo, R. Umer, A. Vascotto, M. Veronese, R. Visintini, M. Zaccaria, D. Zangrando, and M. Zangrando, Nat. Photonics 6, 699 (2012).

2. E. Allaria, D. Castronovo, P. Cinquegrana, P. Craievich, M. Dal Forno, M. B. Danailov, G. D'Auria, A. Demidovich, G. De Ninno, S. Di Mitri, B. Diviacco, W. Fawley, M. Ferianis, E. Ferrari, L. Froehlich, G. Gaio, D. Gauthier, L. Giannessi, R. Ivanov, B. Mahieu, N. Mahne, I. Nikolov, F. Parmigiani, G. Penco, L. Raimondi, C. Scafuri, C. Serpico, P. Sigalotti, S. Spampinati, C. Spezzani, M. Svandrlik, C. Svetina,
M. Trovo, M. Veronese, D. Zangrando, and M. Zangrando, Nat. Photonics 7, 913 (2013).

3. M. Ferianis, A. Borga, A. Bucconi, L. Pavlovic, M. Predonzani, and F. Rossi, in Proceedings of the 33rd International Free Electron Laser Conference (SINAP, 2012), FROAI2.

4. G. Penco, E. Allaria, L. Badano, P. Cinquegrana, P. Craievich, M. Danailov, A. Demidovich, R. Ivanov, A. Lutman, and L. Rumiz, J. Instrum. 8, 05015 (2013).

5. S. Di Mitri, E. Allaria, L. Badano, C. Bontoiu, M. Cornacchia, P. Craievich, M. Danailov, G. DeNinno, B. Diviacco, O. Ferrando, S. Ferry, F. Iazzourene, S. V. Milton, G. Penco, S. Spampinati, M. Trovo', M. Veronese, W. Fawley, S. Lidia, G. Penn, J. Qiang, K. G. Sonnad, M. Venturini, R. Warnock, A. A. Zholents, I. V. Pogorelov, M. Borland, G. Bassi, J. A. Ellison, K. Heinemann, R. Fiorito, and A. Shkvarunets, Nucl. Instrum. Methods Phys. Res. A 608, 19 (2009).

6. P. Schmüser, M. Dohlus, J. Rossbach, and C. Behrens, Springer Tracts in Modern Physics. Vol. 258 (Springer, 2014).

7. R. Bonifacio, N. Narducci, and C. Pellegrini, Opt. Commun. 50, 373 (1984).

8. J. Madey, J. Appl. Phys. 42, 1906 (1971).

9. K. Kondratenko and E. Saldin, Part. Accel. 10, 207 (1980).

10. B. Girard, Y. Lapierre, J. M. Ortega, C. Bazin, M. Billardon, P. Elleaume, M. Bergher, M. Velghe, and Y. Petroff, Phys. Rev. Lett. 53, 2405 (1984).

11. L. H. Yu, Phys. Rev. A 44, 5178 (1991).

12. L. Yu, Nucl. Instrum. Methods Phys. Res. A 393, 96 (1997).

13. G. Penco, M. Danailov, A. Demidovich, E. Allaria, G. De Ninno, S. Di Mitri, W. M. Fawley, E. Ferrari, L. Giannessi, and M. Trovó, Phys. Rev. Lett. 112, 044801 (2014).

14. FERMI Conceptual Design Report, Sincrotrone Trieste internal publication, $S T / F-T N-07 / 12$, https://www.elettra.tries te.it/it/lightsources/fermi/fermi-machine/fermicdr.html.

15. E. Allaria, G. De Ninno, S. Di Mitri, W. M. Fawley, E. Ferrari, L. Frolich, G. Penco, P. Sigalotti, S. Spampinati, C. Spezzani, and M. Trovò, Phys. Rev. ST Accel. Beams 17, 010704 (2014).

16. P. Craievich, S. Di Mitri, M. Milloch, G. Penco, and F. Rossi, Phys. Rev. ST Accel. Beams 16, 090401 (2013).

17. J. Byrd, L. Doolittle, A. Ratti, J. W. Staples, R. Wilcox, M. Stettler, G. D'Auria, M. Ferianis, M. Milloch, and A. Rohlev, in Proceedings of the 22nd Particle Accelerator Conference (IEEE, 2007), WEPMN116.

18. M. Ferianis, in IEEE Proceedings of the 23rd International Linear Accelerator Conference (ORNL, 2006), WE3GRI02.

19. J. M. Byrd, L. Doolittle, A. Ratti, J. W. Staples, and R. Wilcox, in IEEE Proceedings of the 23rd International Linear Accelerator Conference (ORNL, 2006), THP007.

20. J. Kim, J. A. Cox, J. Chen, and F. X. Katner, Nat. Photonics 2, 733 (2008).

21. SIROCCO XS blown fiber system, prysmiangroup.com/ PRYSMIAN GROUP.

22. T. R. Schibli, J. Kim, O. Kuzucu, J. T. Gopinath, S. N. Tandon, G. S. Petrich, L. A. Kolodziejski, J. G. Fujimoto, E. P. Ippen, and F. X. Kaertner, Opt. Lett. 28, 947 (2003).

23. EUROFEL: FP6 Design Study project, contract number: 011935; coordinator: Deutsches Elektronen-Synchrotron (DESY); http://www.eurofel.org.

24. B. Steffen, S. Casalbuoni, P. Schmuser, S. Simrock, M. Tonutti, A. Winter, T. Korhonen, T. Schilcher, V. Schlott, H. Sigg, and D. Suetterlin, in Proceedings of EPAC 2004 (2004), TUPOS06. 
25. A. Winter, F. Lohl, F. Ludwig, H. Schlarb, B. Schmidt, and P. Schmuser, in Proceedings of EPAC 2006 (2006), TUPCH028.

26. G. A. Loew and O. H. Altenmueller, SLAC PUB-135, Aug. 1965.

27. I. Ben-Zvi, J. X. Qiu, and X. J. Wang, in Proceedings of 17th Particle Accelerator Conference (1997).

28. M. Veronese, R. Appio, P. Craievich, and G. Penco, Phys. Rev. Lett. 110, 074802 (2013).

29. H. Loos, T. Borden, P. Emma, J. Frisch, and J. Wu, in Proceedings of PACO7 (2007).

30. R. Appio, "Radiazione coerente di diffrazione da un gap in guida d'onda, prodotta da fasci ultracorti di elettroni nell'acceleratore FERMI@Elettra", Thesis (Univ. of Trieste, 2008).

31. O. Grimm, TESLA-FEL. Report 2008-05.

32. F. Lohl, V. Arsov, M. Felber, K. Hacker, W. Jalmuzna, B. Lorbeer, F. Ludwig, K.-H. Matthiesen, H. Schlarb, B. Schmidt, P. Schmüser, S. Schulz, J. Szewinski, A. Winter, and J. Zemella, Phys. Rev. Lett. 104, 144801 (2010).

33. L. Pavlovic, A. O. Borga, M. Ferianis, M. Predonzani, and F. Rossi, in Proceedings of the Beam Instrumentation Workshop (2010), TUPSM086.

34. A. O. Borga, R. De Monte, M. Ferianis, L. Pavlovic, and M. Predonzani, in Proceedings of the Beam Instrumentation Workshop (2010), TUPSM025.

35. E. Ferrari, E. Allaria, P. Cinquegrana, M. Ferianis, L. Froelich, L. Giannessi, G. Penco, M. Predonzani, F. Rossi,
P. Sigalotti, and M. Veronese, in Proceedings of the IBIC'13 (2013), TUAL1.

36. D. Alesini, G. Di Pirro, L. Ficcadenti, A. Mostacci, L. Palumbo, J. Rosenzweig, and C. Vaccarezza, Nucl. Instrum. Methods Phys. Res. A 568, 488 (2006).

37. M. Rohrs, C. Gerth, H. Schlarb, B. Schmidt, and P. Schmüser, Phys. Rev. ST Accel. Beams 12, 050704 (2009).

38. Y. Ding, C. Behrens, P. Emma, J. Frisch, Z. Huang, H. Loos, P. Krejcik, and M.-H. Wang, Phys. Rev. ST Accel. Beams 14, 120701 (2011).

39. P. Craievich, S. Biedron, C. Bontoiu, S. Di Mitri, M. Ferianis, M. Veronese, M. Petronio, D. Alesini, and L. Palumbo, in Proceedings of the FEL10 Conference (2012), WEPB43.

40. P. Craievich, M. Petronio, S. G. Biedron, D. Castronovo, M. Dal Forno, S. Di Mitri, N. Faure, D. La Civita, G. Penco, L. Rumiz, L. Sturari, R. Vescovo, and D. Wang, IEEE Trans. Nucl. Sci. 62, 120705 (2015).

41. C. Behrens, F.-J. Decker, Y. Ding, V. A. Dolgashev, J. Frisch, Z. Huang, P. Krejcik, H. Loos, A. Lutman, T. J. Maxwell, J. Turner, J. Wang, M.-H. Wang, J. Welch, and J. Wu, Nat. Commun. 5, 3762 (2014).

42. P. Cinquegrana, S. Cleva, A. Demidovich, G. Gaio, R. Ivanov, G. Kurdi, I. Nikolov, P. Sigalotti, and M. B. Danailov, Phys. Rev. ST Accel. Beams 17, 040702 (2014).

43. P. Sigalotti, P. Cinquegrana, A. Demidovich, R. Ivanov, I. Nikolov, G. Kurdi, and M. B. Danailov, Proc. SPIE 8778, 87780Q (2013). 